\title{
Multichannel Photomultiplier for Multipass Thomson Scattering Diagnostics
}

\author{
M. J. R. Monteiro, M. Machida, A. M. Daltrini, and L. A. Berni ${ }^{1}$ \\ Instituto de Física "Gleb Wataghin", Universidade Estadual de Campinas, \\ C. P. 6165, 13083-970, Campinas, SP, Brazil \\ ${ }^{1}$ LAP/INPE-São José dos Campos, SP, Brazil
}

Received on 26 June, 2001

\begin{abstract}
The application of a new multichannel 64 parallel amplification 10 stage photomultiplier arranged in $8 \times 8$ matrix of $20.32 \mathrm{~mm} \times 20.32 \mathrm{~mm}$, detector XP1752 from Philips, for laser light scattering diagnostics is now under development. Signal intensity calibration curve for eight parallel and individual channels, as well as cross talk between channels was measured. Also, Rayleigh scattering on nitrogen gas, normally used as optical calibration method for electron density measurements in Thomson scattering was realized, and the intensity of both detectors was compared making measurements with a tungsten lamp.
\end{abstract}

\section{Introduction}

Thomson scattering is often used to measure the local electron temperature, [1,2] and electron density can be determined if calibration is done by use of Rayleigh scattering [3,4]. For this diagnostic method, one of the critical issues is the very low scattered light signal, which can be increased by use of a higher laser power or by passing the laser beam many times through the same region in the plasma, known as multipass system [5-7].

In our experiments, we use a $3 \mathrm{~J}$ ruby laser, $40 \mathrm{~ns}$ pulse width, and a multipass configuration with one lens and two concentrical spherical mirrors to increase the incident power and, consequently, the scattered signal.

Through the width of this Thomson scattered profile, we can measure the electron temperature. But, when a single photomultiplier is used, one need many pulses of the laser beam as well as many discharges of our tokamak NOVA-UNICAMP[8], loosing therefore quickness and accuracy in our measurements.

So, the purpose to use a multichannel photomultiplier in Thomson scattering is to allow online measurement of the electron temperature and density, with one shot of the laser beam and one discharge of the tokamak.

Also, we can obtain radial information, though the spatial resolution of the detector, improving therefore the accuracy in the measurements and knowledge of the plasma in our machine.

\section{Experimental setup}

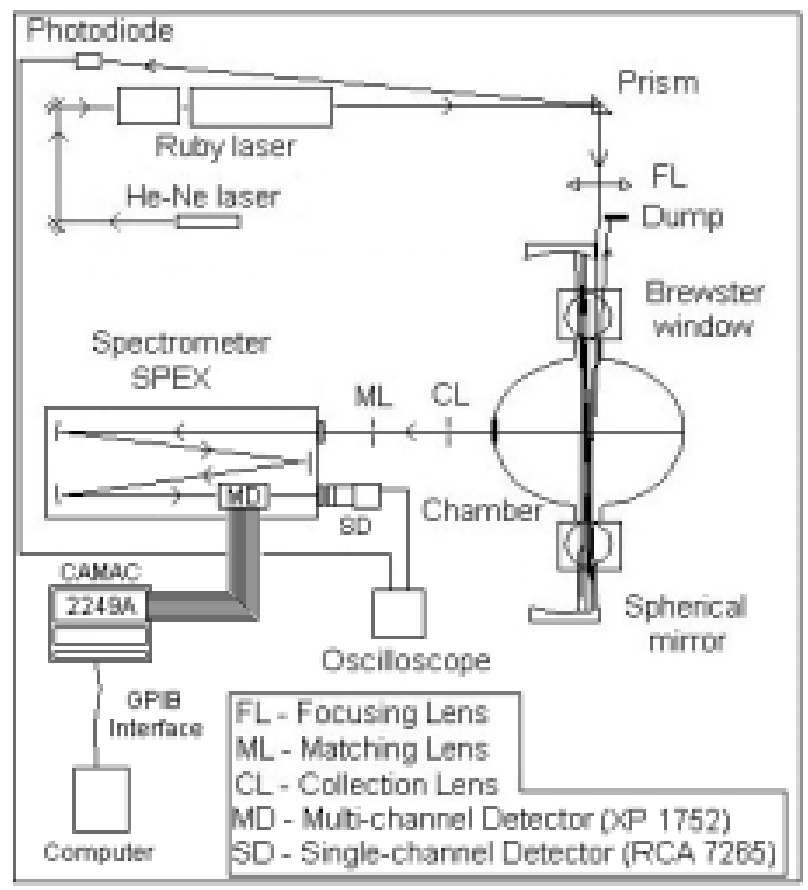

Figure 1. Experimental set up.

In Fig. 1, we can see the experimental set up used for our measurements. The laser beam, after many passages through the central region, it is collected in a beam dump. For the alignment, we used two He-Ne lasers, one to align the ruby laser, and the other to align the collection optics. 
The spectrometer SPEX has $75 \mathrm{~cm}$ focal distance, $11 \AA / \mathrm{mm}$ dispersion relation, and permits to use two detectors. By rotation of a internal mirror we can select, the single channel detector, photomultiplier RCA 7265 , or the multichannel photomultiplier, XP 1752.

Each channel of XP1752 detector has $2.54 \mathrm{~mm} x$ $2.54 \mathrm{~mm}$ of sensitive area, spectral resolution of approximately $27 \AA, 5.0 \mathrm{~ns}$ response time, 10 stage dynodes with gain of $1 \times 106$ at $1150 \mathrm{~V}$, and allows to measure FWHM of Gaussian shape scattered light from $8 \mathrm{~nm}$ ( $6 \mathrm{eV})$ to $80 \mathrm{~nm}(600 \mathrm{eV})$.

The single channel photomultiplier has a response time of $3.5 \mathrm{~ns}, 14$ stage dynodes, and gain of $2 \times 10^{6}$ at $1800 \mathrm{~V}$.

The signals from the detector is taken by analog to digital converters, ADC, LeCroy 2249A and controlled with GPIB to a PC, providing on line measurements of scattered light variation by wavelengths.

\section{Results}

The 64 channels have been set to 8 parallel column outputs. Each column output covers all spectrometer slit height, and one spectral width of $27 \AA$. Signal intensity calibration curves for this eight parallel channels was measured, in the range of $620 \mathrm{~nm}$ to $642 \mathrm{~nm}$, scanning a He-Ne laser, Fig. 2.

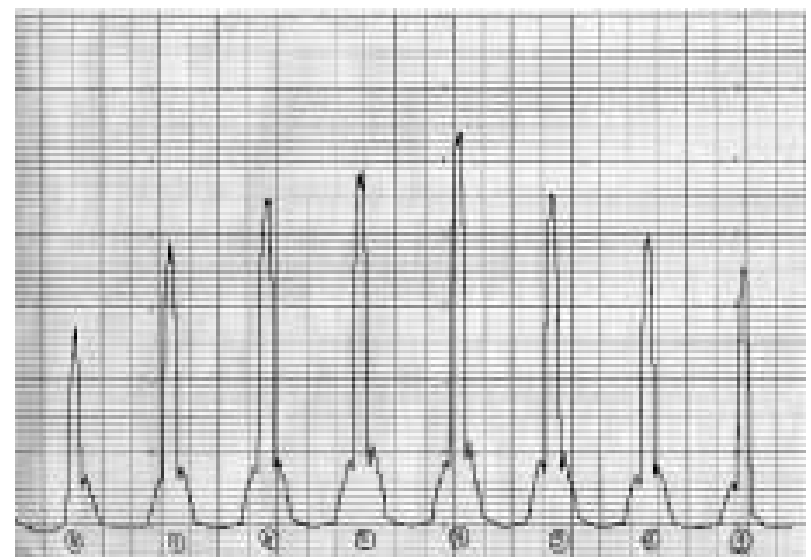

Figure 2. Intensity calibration curves of the 8 parallel spectral channels, 1 to 8 , from right to left, of the multichannel detector XP 1752 .

The cross talk between channels is defined as the influence of signal in one channel caused by adjacent channel. If we observe the right and left sides of the Fig. 2, column channels 1 and 8 respectively, we see that the straight line at the bottom of signals is due to the lack of adjacent channels. So, the influence of one channel on the adjacent channel appears as the small area surrounding the characteristic area of the signal in the channel. So, the cross talk is the ratio between the areas of the influence signal, A1, and characteristic signal, A2, Fig. 3.

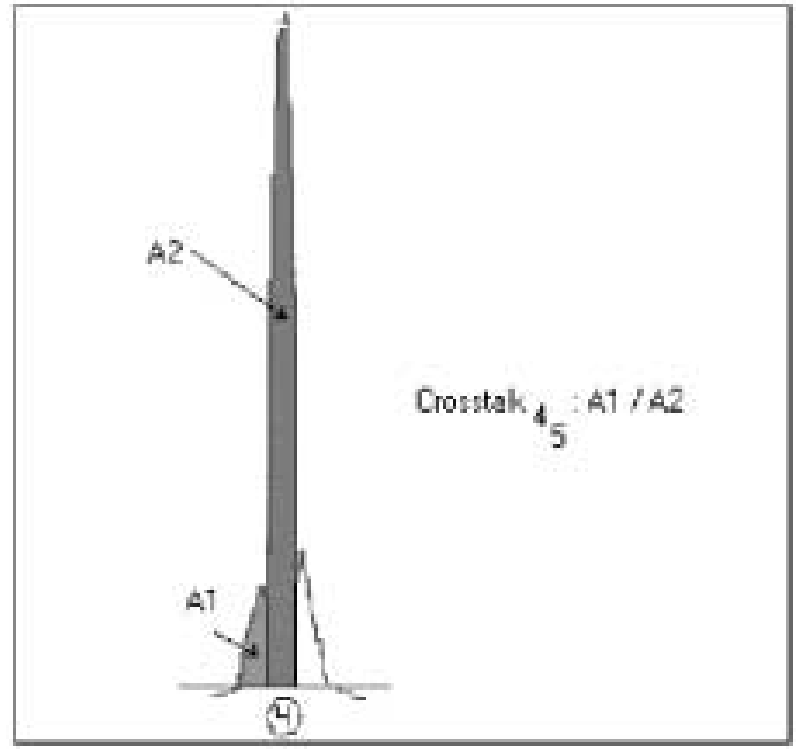

Figure 3. Cross talk caused in the channel 4 by the channel 5.

Measurements using He-Ne laser, slit aperture of $100 \mathrm{~mm}$, show that the mean cross talk between channel is about $14 \%$.

For more precise characterization of the multichannel photomultiplier, intensity calibration of the 64 individual channels was also measured, Fig. 4.

In Fig. 4, each spectral channel has $27 \AA$ resolution and each spatial channel covers $2.5 \mathrm{~mm}$ slit height with $100 \mathrm{~mm}$ slit aperture of the spectrometer. We notice that there is 5.4 intensity variation between channel $(4,5)$ and channel $(1,8)$ which is in accordance to other measurements $[9,10]$.

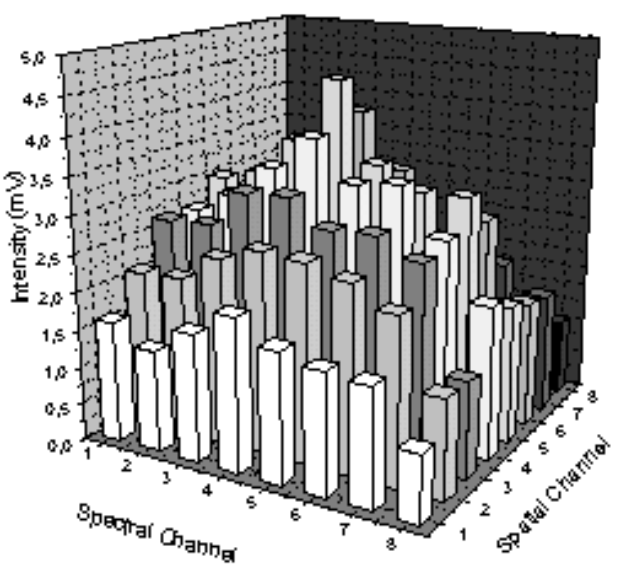

Figure 4. Intensity calibration for the 64 individual channels of the multichannel photomultiplier XP 1752 .

For slit height measurement capability, spatial definition, we measure the intensity variation of the eight 
individual detectors in the spectral channel 4, as function of the slit height, Fig. 5. Again, we use a He-Ne laser and the slit aperture of $100 \mathrm{~mm}$. Notice that for full slit height opening $2.5 \times 8$, we reproduce the spectral channel 4 of figure 4 .

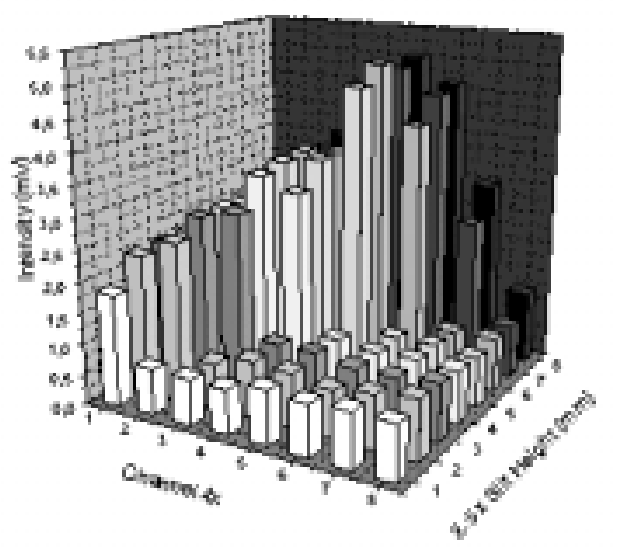

Figure 5. Intensity variation of the spectral channel-4 eight detector as function of the slit height.

Rayleigh scattering measurements on nitrogen gas, normally used as optical calibration method for electron density measurements in Thomson scattering, was done to test the multichannel detector, Fig.6.

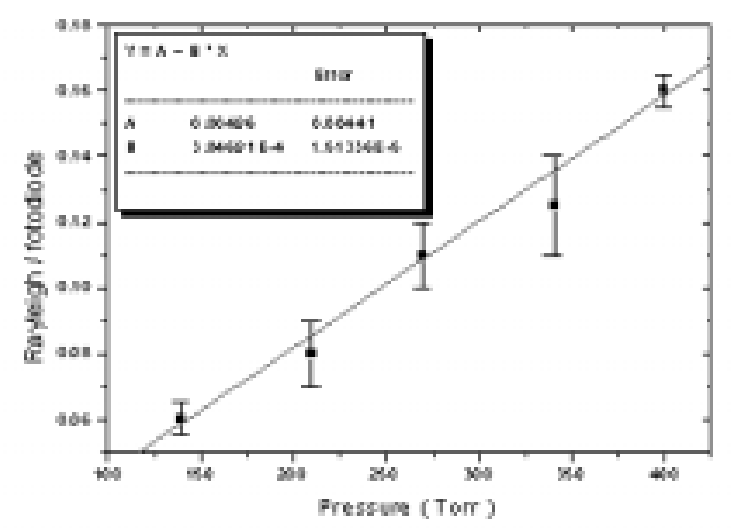

Figure 6. Calibration curve for Rayleigh scattering on nitrogen gas using one spectral column channel (channel 6) of the multichannel photomultiplier.

As we can see, the approximately linear variation of the signals with the pressure is characteristic of the calibration curve for Rayleigh scattering.

Finally, using a tungsten lamp and the same experimental set up, we measure the intensities ratio of the signals at characteristic values of working voltage for single $(1800 \mathrm{~V})$ and multichannel detector $(4,5)(1400 \mathrm{~V})$ photomultipliers, Fig. 7.

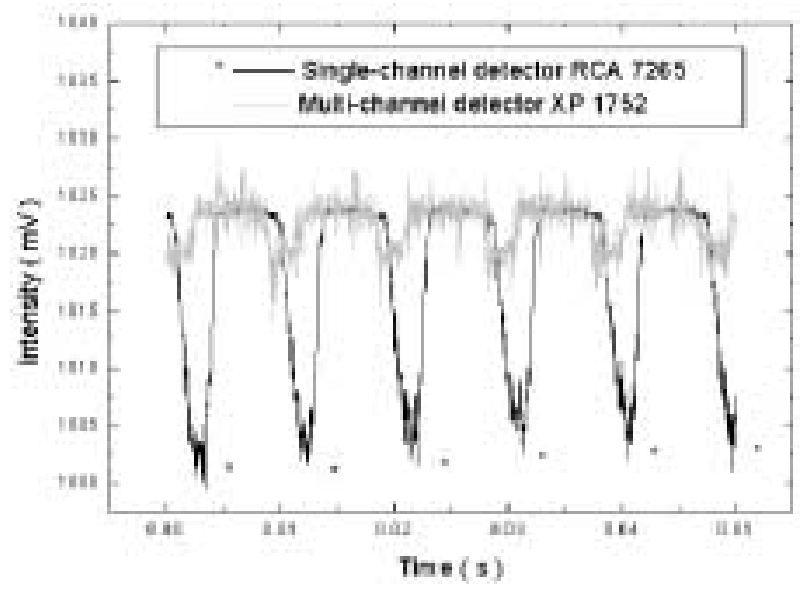

Figure 7 . Intensity measurements for single and multichannel photomultipliers using a tungsten lamp, notice that the ratio is about five times.

\section{Conclusions}

Characterization of the multichannel detector XP 1752 has been done with the purpose of use on Thomson scattering measurements. This detector provides both spectral and spatial resolutions, and same range of intensity compared to single photomultiplier, which should contribute to accuracy and quickness on the local electron temperature and density measurements.

\section{Acknowledgements}

The authors would like to thank FAPESP and FINEP for the financial support.

\section{References}

[1] M.Bassan, R. Bilato, L. Giudicotti, R. Pasqualoto, and A. Sardella, Rev. Sci. Instrum. 68, 718 (1997).

[2] A. Cte, D. Michaud, N. Richard, R. Neufeld, and C.Legros, Rev. Sci. Instrum. 66, 487 (1995).

[3] L. A. Berni, D. O. Campos, M. Machida, S. A. Moshkalyov and S. V. Lebedev; Brazilian Journal of Physics, 26, 755 (1996).

[4] D. G. Nilson, D. N. Hill, J. C. Evans, T. N. Calrstrom, C. L. Hsieh and R. E. Stockdale; Rev. Sci. Instrum. 68, 704 (1997).

[5] M. Yu. Kantor and D. V. Kouprienko; Rev. Sci. Instrum. 70, 780 (1999).

[6] M. Yu. Kantor; Technical Physics Letters, 23, 671 (1997).

[7] M. Yu. Kantor; Technical Physics Letters, 25, 860 (1999).

[8] M. Fukao, K. Mori, T. Tanihara; Plasma Phys. Control. Fusion, 33, 199 (1991).

[9] J. P. Boutot, P. Lavoute and G. Eschard; IEEE Trans. Nucl. Sci., 34, 449 (1987).

[10] L. Eriksson et al.; IEEE Trans. Nucl. Sci., 34, 344 (1987). 